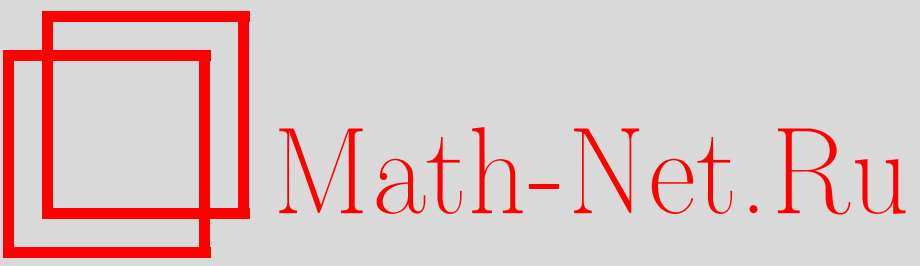

В. В. Белокуров, Ю. П. Соловьев, Е. Т. Шавгулидзе, Об одном методе суммирования расходящихся рядов, УМH, 1999, том 54, выпуск 3, 153-154

DOI: https://doi.org/10.4213/rm156

Использование Общероссийского математического портала Math-Net.Ru подразумевает, что вы прочитали и согласны с пользовательским соглашением

http://www.mathnet.ru/rus/agreement

Параметры загрузки:

IP: 54.81 .137 .203

26 апреля 2023 г., 10:01:43 


\title{
ОБ ОДНОМ МЕТОДЕ СУММИРОВАНИЯ РАСХОДЯШИХСЯ РЯДОВ
}

\author{
В. В. БЕлОКУРОв, Ю. П. СОлОвьЕВ, Е. Т. ШАвГУЛИДЗЕ
}

В настоящей работе предлагается метод суммирования расходящихся рядов, которые могут быть представлены как ряды Тейлора гладких вполне монотонных функций. Стандартным методом суммирования таких рядов является метод Бореля и его модификации [1]. Однако этот метод требует знания всех членов ряда и не позволяет восстановить значение суммы по конечному числу членов ряда, которое обычно только и бывает известно в реальных задачах. Рассматриваемый нами подход является развитием метода вычисления функциональных интегралов с помощью аппроксимации их сходящимися рядами [2]-[4].

Для гладкой вполне монотонной на полупрямой $[0, \infty)$ функции $f(t)$ по теореме С.Н. Бернштейна (см., например, [5]) сушествует такая неотрицателная мера Радона $\mu$, что

$$
f(t)=\int_{0}^{\infty} e^{-t x} \mu(d x)
$$

Положим

$$
A_{2 n}(R)=\frac{(-1)^{n}}{2 \pi} \int_{-R}^{+R} d \rho \int_{-\infty}^{+\infty} d r \exp \left\{-i \rho r-r^{4}\right\} \rho^{2 n}, \quad B_{n}=\int_{0}^{\infty} x^{n / 2} \mu(d x) .
$$

Теорема 1. Пусть $f \in C^{\infty}[0, \infty)$ - вполне монотонная функция на полупрямой $[0, \infty)$, удовлетворяющая условиям $\left|f^{(n)}(0)\right| \leqslant C n^{(4-2 \lambda) n}$, әде Cu $и$ - некоторые положительные постоянные.

Тогда для любого $t>0$ и любого $\varepsilon>0$ найдутся такие $R=R(\varepsilon)$ u $N_{0}=N_{0}(t, \varepsilon, R)$, что при всех $N>N_{0}$

$$
f(t)=\sum_{n=0}^{N} \frac{1}{(2 n) !} t^{n / 2} A_{2 n}(R) B_{n}+\Delta(t, R, N),
$$

əде $|\Delta(t, R, N)| \leqslant \varepsilon$.

ЗАмЕчАНИЕ. В качестве $R$ и $N_{0}$ могут быть взяты любые числа, удовлетворяющие условиям $R \geqslant(\ln (4 f(0) / \varepsilon))^{4 / 5}, N_{0} \geqslant \max \left\{\left(2 t^{1 / 2} R^{2}\right)^{1 / \delta}, \ln 4 C \varepsilon, t^{1 / 4} R^{3 / 2}\right\}$.

Для практического применения теоремы 1 к суммированию расходящихся рядов необходимо еще указать алгоритм вычисления входящих в формулу (1) коэффициентов $B_{n}$. Для четных $n$ $B_{n}=(-1)^{n / 2} f^{(n / 2)}(0)$. Основу метода приближенного вычисления коэфффициентов с нечетными индексами $n$ составляет

Теорема 2. Пусть $f \in C^{\infty}[0, \infty)$ - вполне монотонная функиия на полупрямой $[0, \infty)$. Предположим, что

$$
\left|f^{(n)}(0)\right| \leqslant K^{n} n^{2 n}
$$

где $K$ - некоторое число, большее единиць.

Тогда для любих вещественных $\lambda>0$ и $\varepsilon>0$ найдутся такое натуральное число $N=N(K, \lambda, \varepsilon)$ и такие коэффициенты $b_{m}=b_{m}(K, \lambda, \varepsilon)$, что

$$
\int_{0}^{+\infty} x^{\lambda / 2} \mu(d x)=\sum_{m=0}^{N} b_{m} f^{(m)}(0)+\delta
$$

əде $|\delta| \leqslant \varepsilon$.

Работа выполнена при поддержке Российского фонда фундаментальных исследований (гранты №o. 97-01-00228, 96-15-96030, 96-15-96142). 
Коэффициенты $b_{m}$ вычисляются с помощью равенства

(4) $\int_{0}^{+\infty} x^{n+\frac{\alpha-1}{2}} \mu(d x)$

$$
=\frac{1}{C(\alpha, n)} \int_{0}^{\infty}\left[\int_{0}^{\infty}\left(\cos \left(\xi x^{1 / 2}\right)-\sum_{k=0}^{n-1} \frac{(-1)^{k}}{(2 k) !} \xi^{2 k} x^{k}\right) \mu(d x)\right] \xi^{-(2 n+\alpha)} d \xi
$$

где

$$
C(\alpha, n)=\frac{(-1)^{n} \pi}{2 \cos \frac{\pi \alpha}{2} \Gamma(2 n+\alpha)}, \quad|\alpha|<1 .
$$

Из теорем 1 и 2 вытекает, что значение функции $f(t)$ можно восстановить с любой точностью, используя конечное число членов ряда. А именно, имеет место следующее утверждение.

Теорема 3. При произвольных значениях параметров $K>0, \varepsilon>0 u 0 \leqslant t \leqslant T$ найдутся натуральное число $M=M(K, \varepsilon, T)$ и такой набор коэффициентов $c_{m}=$ $c_{m}(K, \varepsilon, t), 1 \leqslant m \leqslant M$, что для любой функции $f(t)$, удовлетворяющей условиям теоремы 2, выполняется неравенство

$$
\left|f(t)-\sum_{m=0}^{M} c_{m} f^{(m)}(0)\right|<\varepsilon .
$$

Предложенным методом может быть просуммирован, в частности, степенной ряд

$$
\sum_{n=0}^{\infty} \frac{(-1)^{n}}{n !} u_{n} t^{n}
$$

коэффициенты которого образуют две позитивные последовательности $\left\{u_{0}, u_{1}, \ldots\right\},\left\{u_{1}, u_{2}, \ldots\right\}$ и удовлетворяют неравенствам: $0 \leqslant u_{n} \leqslant K^{n}(2 n)$ !.

В этом случае ряд (5) является рядом Тейлора некоторой гладкой вполне монотонной функции $f(t)\left(u_{n}=(-1)^{n} f^{(n)}(0)\right)$ [6], причем эта функция единственна [1].

\section{СПИСОК ЛИТЕРАТУРЫ}

[1] Харди Г. Расходяшиеся ряды. М.: ИЛ, 1951. [2] Belokurov V.V., Shavgulidze E.T., Solovyov Yu.P. // Modern Phys. Lett. A. 1995. V. 10. №39. P. 3033-3041. [3] Белокуров В. В., Соловьев Ю. П., Шавгулидзе Е. Т. // Фунд. прикл. матем. 1997. Т. 3. № 3. С. 693-713. [4] Белокуров В.В., Соловьев Ю. П., Шавгулидзе Е. Т. // УМН. 1997. Т. 52. № 2. С. 153-154. [5] Эдвардс Э. Функциональньй анализ. М.: Мир, 1969. [6] Крейн М. Г., Нудельман А. А. Проблема моментов Маркова и экстремальные задачи. М.: Наука, 1973.

Московский государственный

E-mail: belokur@rector.msu.ru solo@difgeo.math.msu.ru shav@nw.math.msu.ru 